\title{
Square Cylinder Large Eddy Simulation Based on Random Inlet Boundary Condition
}

\author{
Z. Liu \\ School of Civil Engineering, University of Nottingham, Nottingham, Nottinghamshire, NG2 2RD, UK \\ Email:liu_zhe_1213@hotmail.com
}

(Received October 5, 2008; accepted May 20, 2009)

\begin{abstract}
Due to the geometrical similarity to many engineering structures, the flow characteristics around the square cylinder have been studied for some time using the experimental or Computational Fluid Dynamics (CFD) technique. However in the past research the inlet boundary is assumed to be steady for simplifying the simulation model, which is conflict with real flow field. To overcome such problem, in this paper, the time varying inlet boundary condition based on autoregressive moving average (ARMA) model is proposed, based on which this inlet boundary generation procedure is introduced. To study the flow characteristics around the square cylinder, a 2D large eddy simulation procedure numerical description is introduced, and different turbulence intensity will be considered at the inlet boundary. The general aerodynamic parameters such as lift coefficient and drag coefficient and velocity component time averaged values along the centre line of the computational domain are discussed. To study the aerodynamic parameters in the frequency domain, the velocity spectrum at different position around the square cylinder will be discussed.
\end{abstract}

Keywords: LES, Random Inlet, ARMA, CFD.

\section{INTRODUCTION}

This instruction gives you guidelines for preparing papers Computational Fluid Dynamics (CFD) applications in studying the flow characteristics of square cylinder had been for a long time, many research based on LES turbulence modelling have been performed, lizuka et al. (1996) studied the flow around a 2D square cylinder using dynamic SGS model; Srinivas et al. (2006) and Wang et al. (1996) performed the LES simulation of high Reynolds number turbulent flow past a square cylinder; Nakayama et al. (2002) used the LES model to study the flow around the square cylinder. Franke et al. (1993), Bouris et al. (1999), Bosch et al. (1998) and Murakami et al. (1995) studied the vortex shedding of the square cylinder based on the LES model respectively. However, the above did not consider the inflow turbulence boundary condition influence and assumed the upwind direction inlet boundary condition to be steady. Actually to the bluff body located in the real wind field, its surrounding flow characteristic is connected with the upwind direction wind flow, which means the upwind direction inflow turbulence should be considered in the simulation. To overcome such problem, different solution methods have been proposed. Smirnov et al. (2003) proposed one method which involves scaling and orthogonal transformation to a continuous field to generate a superposition of harmonic functions, $\mathrm{Li}$ and Wang (2000) presented a random generation technique for fluctuations in a boundary-layer flow, Glaze and Frankel (2003) proposed Weighted Amplitude Wave Superposition (WAWS) spectral representation method. Klein et al. (1999) also introduced an approach based on signal processing modeling through the use of linear non-recursive filters.

In this paper, the time varying inlet boundary technique based on Autoregressive Moving Average (ARMA) model will be introduced, and the procedure will be described which can be applied in the commercial code ANSYS_CFX. To capture turbulence variation characteristics with time and space 2D LES model will be proposed. The square cylinder is selected as the analysis target. Different turbulence intensity inflow boundary condition will be applied to the target to compare their influence on the flow around the square cylinder. Some conclusions based on the analysis result will be drawn in the end.

\section{THEORY FORMULATION}

The Navier-Stokes equations of continuity and the momentum equations for an incompressible fluid take can be written as: 


$$
\begin{aligned}
& \frac{\partial u_{i}}{\partial x_{i}}=0 \\
& \frac{\partial u_{i}}{\partial t}+\frac{\partial u_{i} u_{j}}{\partial x_{j}}=-\frac{1}{\rho} \frac{\partial p}{\partial x_{i}}+\frac{1}{\operatorname{Re}} \frac{\partial^{2} u_{i}}{\partial x_{j} \partial x_{j}}
\end{aligned}
$$

To LES, the large eddies are resolved in the calculation while the small eddies are modeled based on subgrid scale model (SGS model), so the velocity field can be separated into a resolved part and sub-grid part, the resolved part represent the large eddies, and the subgrid part represents the small scales which are modeled by SGS model. So this process can be realized by the convolution of a function with a filtering $\operatorname{kernel} G$, which includes Gaussian, top-hat or box filtering kernel, here top-hat filtering kernel is used:

$$
\bar{u}_{i}(\vec{x})=\int G(\vec{x}-\vec{\xi}) u(\vec{\xi}) d \vec{\xi}
$$

The velocity $u_{i}$ can be decomposed into the resolved scale part $\bar{u}_{i}$ and the subgrid scale part $u_{i}^{\prime}$.

$$
u_{i}=\bar{u}_{i}+u_{i}^{\prime}
$$

In general LES adopts volume averaging as the filter, so Eq.5.2.1--5.2.2 can be rewritten as:

$$
\begin{aligned}
& \frac{\partial \bar{u}_{i}}{\partial x_{i}}=0 \\
& \frac{\partial \bar{u}_{i}}{\partial t}+\frac{\partial \bar{u}_{i} \bar{u}_{j}}{\partial x_{j}}=-\frac{1}{\rho} \frac{\partial \bar{p}}{\partial x_{i}}+\frac{1}{\operatorname{Re}} \frac{\partial^{2} \bar{u}_{i}}{\partial x_{j} \partial x_{j}}-\frac{\partial \tau_{i j}}{\partial x_{j}}
\end{aligned}
$$

Where $\tau_{i j}$ is the stress term, $\tau_{i j}=\overline{u_{i} u_{j}}-\bar{u}_{i} \bar{u}_{j}$, based on the Smagorinsky model this term can be written in the form of eddy viscosity

$$
\tau_{i j}-\frac{1}{3} \tau_{k k} \delta_{i j}=-2 v_{S G S} \bar{S}_{i j}
$$

Where $\bar{S}_{i j}=\frac{1}{2}\left(\frac{\partial \bar{u}_{i}}{\partial x_{j}}+\frac{\partial \bar{u}_{j}}{\partial x_{i}}\right)$ is the strain rate tensor based on filtered velocity field. The eddy viscosity $v_{S G S}$ can be expressed as:

$$
v_{S G S}=\left(C_{S} \Delta\right)^{2}|\bar{S}|
$$

Where

$$
|\bar{S}|=\sqrt{2 \overline{S_{i j} \bar{S}_{i j}}}
$$

Where $C_{S}$ is the sub-grid scale stress constant and different from the 3D LES model, in 2D LES, the filtering process is only applied in the two directions, so $\Delta=(\Delta x \Delta y)^{1 / 2}$ defined by the grid spacing. To approximate the terms in the above equation, many discretization methodologies or procedures can be applied. The SIMPLE algorithm for pressure correction is employed by Patankar and Spalding (1972) and Bouris and Bergeles (1999), in general the first order implicit Euler scheme for time discretisation is more stable than the explicit scheme.

Papadakis and Bergeles (1995) used the fully Bounded Second Order Upwind (BSOU) Scheme to difference the convective terms, with replacing the hybrid central/upwind differencing scheme, Franke and Rodi (1991) presented that the QUICK third-order upwind differencing scheme would introduce over and undershoots due to the unbounded nature of such scheme.

In current study, the central differencing scheme will be adopted to deal with the advection scheme, which is less dissipative and provide good approximation for the answer. To the transient scheme the second order Backward Euler scheme is employed.

\section{DifFiCULTIES IN APPLYING LES INTO CWE}

Although LES model had been applied in the wind engineering of civil engineering, there are still some difficulties in its application in the CWE. Murakami (1998) proposed some problems existing in the CWE. In general Reynolds number of the flow field treated in wind engineering is usually quite large; always more than 108, which requires fine grid resolution, and the Higher Reynolds number, the more gird is needed. Such high Reynolds numbers simulations require much more computer resources than currently available super computers capacities, which is therefore not practicable.

The flow field around a bluff body is very complicated since it is characterized by impinging, separation, vortex shedding, etc. furthermore it is highly threedimensional (3D). Because most of structures in CWE are located in fully development turbulence field and the aerodynamics of these structures are influenced by the upwind direction turbulence, which makes it impossible to use conventional inflow boundary condition with mean value in LES computations. So effective simulating the time varying inlet boundary condition is very important.

Artificial methods for generating turbulent inflow are described later. To consider the inflow turbulence, some techniques for generating velocity fluctuations is required. Several techniques have been invested by researchers and the extended discussion will be presented in section 4 .

\section{DESCRIPTION OF REFERENCES}

The approach of wind velocity fluctuations generation at the inflow boundary condition of LES based on the prescribed spatial correlation and turbulence intensity is one of the most important unresolved issues in CFD research.

Several methods have been proposed to solve such problem. The simplest one to define the inflow 
boundary conditions from RANS data is to neglect the turbulent fluctuations entirely. The velocity field at the LES inlet is then defined by the ensemble-averaged mean profiles from the RANS computations. This means that the incoming flow is laminar in a sense that the velocity does not fluctuate, but the mean profiles of the velocity are still those of a turbulent flow. The second method is to carry out preliminary computation of turbulent flow fields based on LES, and the turbulent flow generated by a turbulence grid settled at the inflow boundary of a computational domain is stored with the time series of velocity fluctuations for inflow boundary conditions.

This method, however, requires a large computational load. The other one is to generate a time series of velocity data by inverse Fourier transform of the appropriate spectral density function with the spectral components randomly distributed phase angle. If $3 \mathrm{D}$ densities are used then the velocity components can be made to satisfy continuity, and this approach has been used by Lee et al. (1992) for a relatively simple turbulent inflow, however it is difficult to define the full 3D densities in the more complex flow typical of a turbulent wind environment. So in the following section, a technique based on ARMA model turbulence generation technique will be described.

\subsection{ARMA Random Inflow Turbulence Generation Technique}

In general, the instantaneous velocity may be described as:

$$
U(x, y, z, t)=\bar{U}(Z)+u(x, y, z, t)
$$

where $\bar{U}(Z)$ is the mean wind speed at height $z$ of point of co-ordinates $x, y, z ; u(x, y, z, t)$ is the velocity fluctuation part with zero mean value, a random function of space $(x, y, z)$ and time $t$. And the wind velocity field is considered as a 'multivariate multidimensional random variable', the power spectral density (PSD) becomes a multidimensional function and assumes the form:

$$
<S(\omega)>_{N_{p} \times N_{p}}=\left(\begin{array}{cccc}
S_{11}(\omega) & S_{12}(\omega) & \cdots & S_{1 N_{p}}(\omega) \\
S_{21}(\omega) & S_{22}(\omega) & \cdots & S_{2 N_{p}}(\omega) \\
\vdots & \vdots & \cdots & \vdots \\
S_{N_{p} 1}(\omega) & S_{N_{p} 2}(\omega) & \cdots & S_{N_{p} N_{p}}(\omega)
\end{array}\right)_{N_{p} \times N_{p}}
$$

Here $N_{P}$ is the number of the points. Many different expressions for the functions in Eq. 11 have been developed over the years and the 'classical' expression is given by:

\section{Cross-spectrum:}

$S_{j i}(\omega)=\sqrt{S_{j j}(\omega) S_{i i}(\omega)} \operatorname{coh}_{j i}(\omega) e^{-\omega \theta_{i j}}$

Coherence:

$$
\begin{aligned}
& \operatorname{coh}_{i j}\left(x_{i}, y_{i}, z_{i} ; x_{j}, y_{j}, z_{j} ; \omega\right) \\
& =\exp \left(-\frac{\omega}{2 \pi} \frac{\sqrt{c_{x}^{2}\left(x_{i}-x_{j}\right)+c_{y}^{2}\left(y_{i}-y_{j}\right)+c_{z}^{2}\left(z_{i}-z_{j}\right)}}{\bar{U}\left(z_{k}\right)}\right)
\end{aligned}
$$

where $\bar{U}\left(z_{k}\right)=\left(\bar{U}\left(z_{i}\right)+\bar{U}\left(z_{j}\right)\right) / 2$ is the mean wind velocity between two points $i$ and $j$ where the average wind speed are $\bar{U}\left(z_{i}\right)$ and $\bar{U}\left(z_{j}\right)$, $\theta_{i j}=x_{i} / \bar{U}\left(z_{i}\right)-x_{j} / \bar{U}\left(z_{j}\right)$ is the time lag (where it is assumed that wind direction coincides with $x$ ) and $c_{x}, c_{y}, c_{z}$ are the decay coefficients.

For ARMA model the velocity component can be described as:

$$
\begin{aligned}
& \{u(t)\}_{N_{P^{\times 1}}}=\sum_{1}^{p}[\varphi(i)]_{N_{P} \times N_{P}}\{u(t-i \Delta t)\}_{N_{P^{\times 1}}}+ \\
& \sum_{1}^{q}[B(i)]_{N_{P} \times N_{P}}\{a(t-i \Delta t)\}_{N_{P} \times 1}
\end{aligned}
$$

which expresses the signal at a given time through a linear combination of the previous events to which a stochastic component ' $a$ ' is added. In Eq.14 index ' $p$ ' refers to the order of AR in the model, while ' $q$ ' is the order of the moving average (MA) component.

$\{a(t-i \Delta t)\}_{N_{P} \times 1}=W N(0, \sigma)$ is white noise signal with 0 and $\sigma$ as average and standard deviation, $\{u(t-i \Delta t)\}_{N_{P^{\times 1}}}$ is the velocities on the different point of the field. It is necessary now to introduce the correlation matrices defined as

$$
\begin{aligned}
& R_{u u}(k \Delta t)=\left[E\left\{u(t) u^{T}(t+\Delta t)\right\}\right]_{N_{p} \times N_{P}} \\
& R_{u a}(k \Delta t)=\left[E\left\{u(t) a^{T}(t+\Delta t)\right\}\right]_{N_{P} \times N_{P}}
\end{aligned}
$$

In the hypothesis of ergodicity, it can be shown that the correlation and the PSD form a Fourier's pair; therefore

$$
R_{u u_{i j}}(\tau)=\frac{1}{2 \pi} \int_{0}^{\infty} S_{i j}(\omega) \cos \left(\omega\left(\tau-\theta_{i j}\right)\right) d \omega
$$

Based on the above equation, given the symmetry of matrix $S$, it follows:

$$
R_{u u_{i j}}(\tau)=R_{u u_{j i}}(-\tau) \rightarrow R_{u u}(\tau)=R_{u u}^{t}(-\tau)
$$

Post-multiplying Eq. 14 with $\left[\left\{u_{r-1}\right\}^{T} \cdots\left\{u_{r-p}\right\}^{T}\right]$, after some transformation, this final system allows us to find the unknowns 


$$
\begin{aligned}
& {\left[\begin{array}{cccc}
{\left[R_{\text {uu }}(0)\right]^{T}} & {\left[R_{\text {uu }}(1)\right]^{T}} & \cdots & {\left[R_{\text {uu }}(p-1)\right]^{T}} \\
{\left[R_{\text {uu }}(-1)\right]^{T}} & {\left[R_{\text {uu }}(0)\right]^{T}} & \cdots & {\left[R_{\text {uu }}(p-2)\right]^{T}} \\
\vdots & \vdots & & \vdots \\
{\left[R_{\text {uu }}(1-p)\right]^{T}} & {\left[R_{\text {uu }}(2-p)\right]^{T}} & \cdots & {\left[R_{\text {uи }}(0)\right]^{T}}
\end{array}\right]\left[\begin{array}{c}
{\left[\varphi_{1}\right]^{T}} \\
{\left[\varphi_{2}\right]^{T}} \\
\vdots \\
{\left[\varphi_{p}\right]^{T}}
\end{array}\right]} \\
& =\left[\begin{array}{c}
{\left[R_{u u}(-1)\right]^{T}} \\
{\left[R_{u u u}(-2)\right]^{T}} \\
\vdots \\
{\left[R_{u u}(-p)\right]^{T}}
\end{array}\right]
\end{aligned}
$$

Once the coefficient's matrices are determined $[\varphi]$ it is possible to estimate matrix $\left[B_{0}\right]$. This is easily shown considering that Eq. 19 can be written as

$$
\left\{u_{r}\right\}=\sum_{i=1}^{p}\left[\varphi_{i}\right]\left\{u_{r-i}\right\}+\left[B_{0}\right]\left\{a_{r}\right\}
$$

Post-multiplying Eq.20 by $\left\{u_{r}\right\}^{T}$ and taking the average, we can get

$$
\left[B_{0}\right]\left[R_{a u}(0)\right]=\left[R_{u u}(0)\right]-\sum_{i=1}^{p}[\varphi(i)]\left[R_{u u}(-i)\right]^{T}
$$

Taking $\left[B_{0}\right]=\left[R_{a u}(0)\right]^{T}$ and assuming $\left[B_{0}\right]$ to be lower triangular it is possible to obtain it from Eq.21 through a Cholesky's decomposition.

\subsection{Major Steps of Velocity Generation}

Based on the proposed theory in the above, 2D LES time varying velocity inlet boundary condition generation procedure can be described as the following: (see, Fig. 1)

1. Given an wind velocity spectrum

2. Generation the wind velocity time history based on the method introduced in section 4.1

2.1 First, the velocity fluctuations are generated in region (1) by considering the four-point $((1,1),(2,1)$, $(1,2),(2,2))$ correlations.

2.2 Next, velocity fluctuations are generated in region 2 , the points $((2,1),(2,2))$ correlations is used as the region (1).

2.3 The same procedure is carried out in region (3) (m).

3 Save the generation wind velocity time history file as the external file

4. Read the wind velocity time history file using the USER Fortran

5. Interpolate every time step velocity profile based on the inlet boundary coordinates

6 . Solve the problem at every time step

\section{Case Studies}

\subsection{Parameters}

In this part of simulations, the block structured grid is used in the whole domain, (Fig. 2a, the boundary condition and fluid domain can be seen in Fig. 2b. the square cylinder model dimension can be seen in Table 1. The parameters about element number and Reynolds number etc can be seen in Table 2. To catch the velocity components turbulence characteristics, to every single simulate case, two monitors points are set in the domain, the position is shown in Table 3. To catch the velocity components turbulence characteristics, three monitor points are set in the domain, the positions are shown in Table 4.

\subsection{Turbulence PSDF}

Power spectrum density function (PSDF) (Figs. 3-5) and coherence functions are given by:

$$
\begin{gathered}
\frac{n S_{u}(M ; n)}{\sigma_{u}^{2}}=\frac{6.868 n L_{u}(M) / U(M)}{\left[1+10.302 n L_{u}(M) / U(M)\right]^{5 / 3}} \\
\frac{n S_{w}(M ; n)}{\sigma_{w}^{2}}=\frac{6.103 n L_{w}(M) / U(M)}{1+63.181\left(n L_{w}(M) / U(M)\right)^{5 / 3}} \\
\operatorname{Coh}\left(M, M^{\prime} ; n\right)=\exp -\left\{\frac{2 n \sqrt{\sum_{r} C_{r \varepsilon}^{2}\left|r-r^{\prime}\right|^{2}}}{U(M)+U\left(M^{\prime}\right)}\right\}
\end{gathered}
$$

$(\varepsilon=u, w)$

Where, $\sigma_{\varepsilon}$ and $L_{\varepsilon}(M)$ are the standard deviation and the integral length scale, respectively, of the turbulence component $\quad \varepsilon(t)=u(t), w(t) \quad ; \quad r=y, z$ and $r=y^{\prime}, z^{\prime}$ are the coordinates of the points $M$ and $M^{\prime}$ along the deck axis; $C_{r \varepsilon}$ is the exponential decay coefficient of $\varepsilon(t)$ along $r$.

Figs. 5 and 6 show the different parameter's power spectrum curve vs. the target spectrum, at the condition, $U_{\text {mean }}=1.0 \mathrm{~m} / \mathrm{s}, u$ and $w$ component turbulence intensity $20 \%$. From these figures, it can be found that the artificially generated velocity spectrum agree with the target spectrum very well.

\section{Results}

\subsection{Mean Inlet Case}

Before discussing the inlet turbulence influence on the flow around the square cylinder, firstly the steady inlet case lift force power spectrum based on 2D LES is shown in Fig. 7. In Fig. 7, it can be found there are two peaks value in the frequency domains, while the first 
peak value is the dominant vortex shedding frequency value.

Fig. 8 and Fig. 9 show the comparison between the different position monitor point velocity components PSD and Von Karman spectrum, it can be found the front position point velocity component PSD is smaller than target spectrum, while the top and back position is larger than the target spectrum; the back point value is the largest one among the values. The reason is that when the flow pass around the bluff body, the flow separate and generates the shear layer near the top surface of the bluff body, there is energy transfer happening at this zone. To the back point value, after the flow pass by the bluff body, strong vortex happens at the back of the bluff body and changes the velocity and transfer the energy.

\subsection{Time Varying Boundary Inlet Cases}

In section 6.1, analysis results considering steady inlet boundary were discussed. To compare different inlet boundary condition influence, the inflow turbulence boundary with time varying is considered in the stationary square cylinder section real model.

\subsubsection{Mean value}

To validate current simulation results, Fig. 10 shows the velocity component time-averaged value along the centre liner under different inlet boundary conditions, and these values are compared with other experimental data from Durao et al. (1988) and Lyn et al. (1995) at Reynolds number 13,000 and 22,000 respectively.

From Fig. 10, it can be found that with the inlet turbulence intensity increasing, dimensionless mean streamwise velocity component $(u)$ value at the front of square cylinder varying is not very clear, while at downwind direction of square cylinder, the value considering the inlet perturbation is larger than the one with smooth inflow. But after some distance from the bluff body, there is no such variation trend. The reason is that after some distance to the square cylinder, the inflow turbulence boundary influence is not mainly dominant factor, while the vortex dominates the flow varying and contributes to the streamwise component varying.

\subsubsection{Velocity components RMS value}

Figure 11 shows the streamwise velocity components ( $u$ ) RMS value along the centre line of the domain under different inlet boundary conditions. It can be found the velocity component varying trends are almost the same under different boundary conditions at the upstream direction of the square cylinder, Comparing the different inlet boundary conditions' results, it can be found that for to the $u$ component RMS value, the $10 \%$ turbulence intensity value at the downstream direction is larger than the other conditions, after some distance the other conditions values are larger than $10 \%$ turbulence intensity value, while to vertical component $(w)$ RMS there is the same varying process.

\subsubsection{Time history value and power spectrum value}

Figures 12 and 13 show the square cylinder drag and lift force time history curves under the different turbulence intensity inlet condition. It can be found the lift and drag force time history curves are randomly distributed and contain much noise peak values, with the inlet turbulence intensity increasing, the amplitudes of curves increase, and the peak values' phase angles also change with the turbulence intensity increasing.

Figure 14 show front monitor point velocity components time history curves. From this figure, it can be found that the velocity components value of the cases considering the turbulence intensity are larger than the one with mean inlet boundary condition. And the curve show much more random characteristics. With the intensity increasing, the value amplitude is also increasing.

To learn the velocity varying characteristics in the frequency domain, Figs. 15 and 16 show the velocity component PSD at the front point position under different inlet boundary conditions.

From the above figures, it can be found the streamwise $(u)$ and vertical $(w)$ velocity component PSD values considering the turbulence intensity agree with the target spectrum-Von Karman velocity spectrum very well, while in the Figs. 15 and 17, the target spectrum is larger than the spectrums of the 2D large eddy simulation with steady inlet, which can further explain that the time varying inlet boundary condition compensate the wind velocity input. And the top and back monitor point velocity component PSD value has the same varying process as the case with smooth inflow condition.

Figure 17 plots the drag and lift force PSD value under different inlet conditions. With the turbulence intensity increasing the lift and drag force PSD value are larger than the one with mean inlet boundary condition. Even for the cases considering different inlet turbulence intensity, with the intensity increasing, the force spectrum is also increasing.

To study the turbulence intensity influence on the flow around the square cylinder, Figs. 18 and 19 show the top and back monitor point velocity component spectrum at different inlet conditions. From these two figures, it can be found that at the top point position, the streamwise velocity component $(u)$ PSD value generated by the mean inlet velocity condition is large than the other three inlet conditions values, which means the inflow turbulence conditions at the $y$ direction change the flow separation and shear layer pattern, absorb the energy transfer from the leeward direction, while to the vertical velocity component $(w)$, this varying process is reversed. The PSD value considering the time varying inlet boundary condition is larger than the one with smooth inflow condition, which figures out the turbulence inlet boundary conditions input much vertical component perturbation. 
To back point position, the streamwise velocity component $(u)$ of the small and medium turbulence intensity PSD values are larger than the values generated by the smooth flow and high turbulence intensity at the high frequency range. This is because the high turbulence intensity increases the streamwise component $(u)$ perturbation, while at the downstream direction, vortex shedding existing change the velocity value and flow pattern. To vertical component $(w)$ PSD value, in Fig. 19, it can be found the smooth flow and small turbulence intensity values are larger than the medium and high turbulence intensity generated value at the high frequency range, which means vortex shedding transfers more energy to the vertical component velocity, while to the medium and high turbulence intensity, they reduce and damp more energy from the vortex shedding. At the same time, we can also find with the turbulence intensity increasing, the velocity component PSD values are also increasing in the whole.

\subsubsection{Instantaneous result}

Numerical simulation results for the instantaneous value for vorticity $\mathrm{Z}$ are presented in this part. Figure 20 shows instantaneous vorticity $\mathrm{z}$ result at the same no dimensional time step under different inlet boundary condition. From Fig. 20, it can be found that in the case of smooth inflow, we can clearly recognize the shear layers separated from frontal corners. This shear layer exists steadily at some distance from the side of a prism, and does not swing so much. It can be observed that the growth of inherent instabilities thickens the mean shear layers, when turbulence is added to the oncoming flow. On the other hand, in the case of turbulent flow, the separated shear layer strongly interacts with the oncoming turbulence. And with the turbulence intensity considered, there is clear waves propagating from the inlet and then interacting with the vortex generated by the separating flow. There is intermittent reattachment of shear layers by higher inflow turbulence.

\subsubsection{General aerodynamic parameters}

Table 5 summarizes the general aerodynamic parameters under different boundary conditions. From this table, it can be found the with the inlet turbulence increasing, the drag coefficient mean value $\left(\overline{\boldsymbol{C}}_{D}\right)$, RMS value $\left(\boldsymbol{C}_{D, m s}\right)$ and lift coefficient RMS value $\left(\boldsymbol{C}_{L, m s}\right)$ increase. This suggests the inlet turbulence influence on the bluff body aerodynamics characteristics again.

\section{CONCLUSIONS}

Based on the above results analysis, the following conclusions can be drawn for this paper.

1. From current simulation results, it can be found $2 \mathrm{D}$ LES can be used to predict the flow characteristics around the bluff body and current inflow turbulence generation method is an effective method to consider the turbulence from the upwind direction.
2. From the drag and lift force time history curves, it can be found the inflow turbulence boundary condition show much more random characteristics, while the smooth inflow one has no such characteristics. The forces acting upon square cylinder bodies are directly related to the flow structure and the formation of vortices at the leading and trailing edge. These vortices strongly interact with each other as well as with the body surfaces.

3. From the data comparison between the mean velocity inlet case and the time varying velocity inlet cases, it can be found that the oncoming flow turbulence will influence the bluff body flow pattern and the separated shear layer strongly interacts with the oncoming turbulence. And from the lift and drag force time history curves, it can be found that the curves considering turbulence inflow condition show much more random characteristics, while to the force spectrum, the turbulence inflow condition will increase the drag and lift force PSD magnitude.

4. From the velocity spectrum comparison, it can be found the at the front point position, the velocity PSD value considering the turbulence inflow condition agree with the target spectrum very well, while the value of smooth inflow is lower than the target spectrum. But at the top and back point position, the value is larger than the target spectrum, which is mainly caused by the shear layer separation and vortex shedding happening.

\section{ACKNOWLEDGEMENTS}

The author acknowledges the support from the "UK/CHINA researcher's scholarship for the excellence" and School of Civil Engineering in University of Nottingham.

\section{REFERENCES}

Iizuka, S., S. Murakami, A. Mochida and S. Lee (1996). Large eddy simulation of turbulent flow past 2D square cylinder using dynamic SGS model. Generation of inflow turbulence LES based on 3D energy spectrum in wave number space. In Proc, AIJ annual meeting, 537-538.

Srinivas, Y., G. Biswas, A.S. Parihar and R. Ranjan (2006). Large eddy simulation of high Reynolds number turbulent flow past a square cylinder. $J$. Engrg. Mech. ASCE 132(3), 327-335.

Wang, G. and S.P. Vanka (1996). Large-eddy simulations of high Reynolds number turbulent flow over a square cylinder. Dept. of Mechanical and Industrial Engineering, Rep. No. CFD 96-02, Univ. of Illinois at Urbana-Champaign, Ill.

Nakayama, A. and S.N. Vengadesan (2002). On the influence of numerical schemes and subgrid-stress models on large eddy simulation of turbulent flow past a square cylinder. International Journal for Numerical Methods in Fluids 38, 227-253. 
Bouris, D. and G. Bergeles (1999). 2D LES of vortex shedding from a square cylinder. J. Wind Eng. Ind. Aerodyn. 80, 31-46.

Bosch, G. and W. Rodi (1998). Simulation of vortex shedding past a square cylinder with different turbulence models. International Journal for Numerical Methods in Fluids 28, 601-616.

Franke, R. and W. Rodi (1993). Calculation of vortex shedding past a square cylinder with various turbulence models, in: U. Schumann et al. (Eds.). Turbulent Shear Flows 8, Springer, Berlin.

Murakami, S. and A. Mochida (1995). On turbulent vortex shedding flow past 2D square cylinder predicted by CFD. J. Wind Eng. Ind. Aerodyn. 54, 191-211.

Glaze, D. and S. Frankel (2003). Stochastic inlet conditions for large-eddy simulation of a fully turbulent jet. AIAA Journal 41(6), 1064-1073.

Klein, M., A. Sadiki and J. Janicka (2003). A digital filter based generation of inflow data for spatially developing direct numerical or larger eddy simulation. J. Comput. Phys. 186, 652- 665.

Li, C.W. and J.H. Wang (2000). Large-eddy simulation of free surface shallow-water flow. Int. J. Numer. Method. Fluids 34, 31-46.
Smirnov, A., S. Shi and I. Celik (2001). Random flow generation technique for large-eddy simulations and particle-dynamics modeling. J. Fluids Engng. 123, 359-371.

Lilly, D.K. (1967). The representation of small scale turbulence in numerical simulation experiments. in: H.H. Goldstine (Ed.), Proc. IBM Scientific Computing Symposium o Environmental Sciences, IBM Form No. 320-1951, 195-210.

Breuer, M. and M. Pourquie (1996). First experiences with LES of flows past bluff bodies. In: W. Rodi. G. Bergeles (Ed.), Proc. 3rd Int. Symp. on Engineering Turbulence Modeling and Measurements, Heraklion-Crete, Greece, Elsevier, Amsterdam, 177-186.

Murakami, S. (1998). Overview of turbulence models applied in CWE-1997. J. Wind. Eng. Ind. Aerodyn. 74-76, 1-24.

Simiu, E. and R.H. Scanlan (1996). Wind effect on structures. John Wiley \& Sons, New York.

Parker, R. and M.C. Welsh (1981). The effect of sound on flow over bluff bodies. University of Wales, Swansea, Mechanical Engineering Report, $\mathrm{MR} / 87 / 81$.

Table 1 Domain dimension

\begin{tabular}{c|c|c|c|c|c|c|c|c|c|}
\hline CASE & $\boldsymbol{B} / \boldsymbol{D}$ & $\boldsymbol{B}(\boldsymbol{m})$ & $\boldsymbol{D}(\boldsymbol{m})$ & $\boldsymbol{H 1}(\boldsymbol{m})$ & $\boldsymbol{H} \mathbf{2}(\boldsymbol{m})$ & $\boldsymbol{H 3}(\boldsymbol{m})$ & $\mathbf{L 1}(\boldsymbol{m})$ & $\mathbf{L 2}(\boldsymbol{m})$ & $\mathbf{L 3}(\boldsymbol{m})$ \\
\hline R1 & 1 & 2 & 2 & 10 & 6 & 20 & 11 & 4 & 11 \\
\hline
\end{tabular}

Table 2 Computational cases parameters

\begin{tabular}{c|c|c|c|c|c|c|c}
\hline CASE & $\boldsymbol{B} / \boldsymbol{D}$ & $\boldsymbol{B}(\boldsymbol{m})$ & $\boldsymbol{D}(\boldsymbol{m})$ & $\begin{array}{c}\text { Inlet Velocity } \\
(\mathbf{m} / \mathbf{s})\end{array}$ & $\begin{array}{c}\text { Reynolds } \\
\text { Number }\end{array}$ & Time Step $(\boldsymbol{s})$ & Total Time (s) \\
\hline R1 & 1 & 2 & 2 & 1 & $1.69 \mathrm{E} 5$ & 0.01 & 600 \\
\hline
\end{tabular}

Table 3 The inlet turbulence parameters

\begin{tabular}{c|c|c|c}
\hline & $\boldsymbol{U}_{\text {mean }}(\mathbf{m} / \mathbf{s})$ & $\boldsymbol{\sigma}_{u}^{2} / I$ & $\boldsymbol{\sigma}_{w}^{2} / I$ \\
\hline V1 & 1 & $10 \%$ & $10 \%$ \\
\hline V2 & 1 & $20 \%$ & $20 \%$ \\
\hline V3 & 1 & $30 \%$ & $30 \%$ \\
\hline
\end{tabular}

Table 4 Monitor point positions for square cylinder

\begin{tabular}{c|c|c}
\hline Front Point & Top Point & Back Point \\
\hline $\mathrm{x}=11 \mathrm{~m}, \mathrm{y}=13 \mathrm{~m}$ & $\mathrm{x}=12 \mathrm{~m}, \mathrm{y}=14 \mathrm{~m}$ & $\mathrm{x}=14 \mathrm{~m}, \mathrm{y}=13 \mathrm{~m}$ \\
\hline
\end{tabular}


Z. Liu / JAFM, Vol. 3, No. 1, pp. 35-45, 2010.

Table 5 General aerodynamic parameters under different inlet boundaries

\begin{tabular}{c|c|c|c}
\hline Inlet & $\overline{\boldsymbol{C}}_{\boldsymbol{D}}$ & $\boldsymbol{C}_{D, r m s}$ & $\boldsymbol{C}_{L, \text { rms }}$ \\
\hline Smooth & 2.01 & 0.249 & 1.02 \\
\hline $\mathbf{I S}=\mathbf{0 . 1}$ & 2.09 & 0.289 & 1.04 \\
\hline $\mathbf{I S}=\mathbf{0 . 2}$ & 2.12 & 0.359 & 1.07 \\
\hline IS=0.3 & 2.19 & 0.434 & 1.12 \\
\hline
\end{tabular}

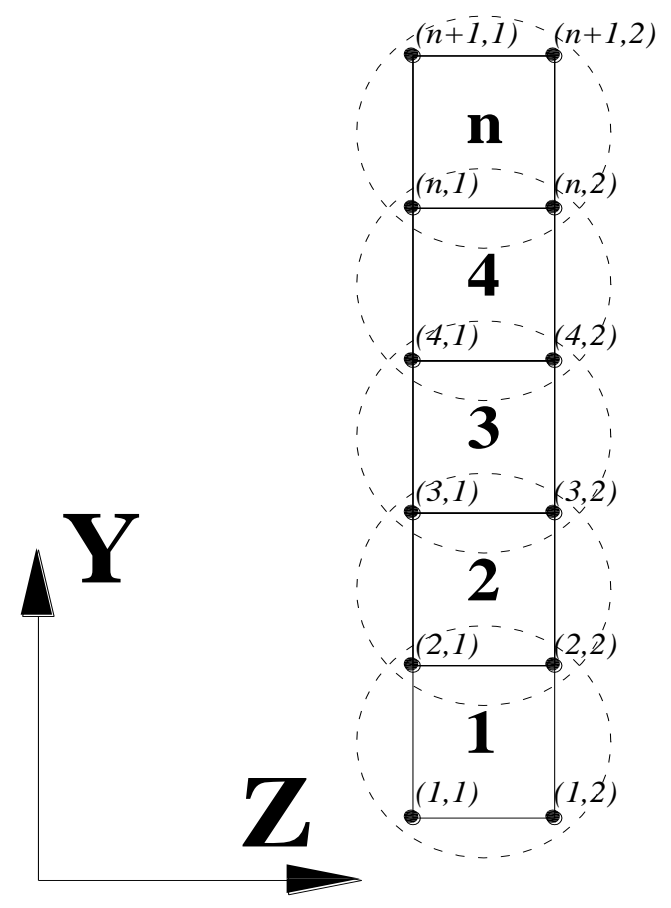

Fig. 1. Interpolate point position

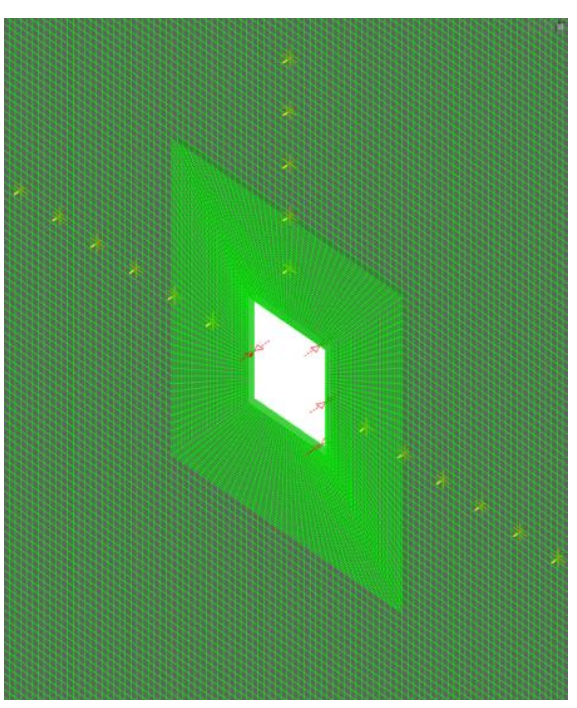

(a) Domain mesh

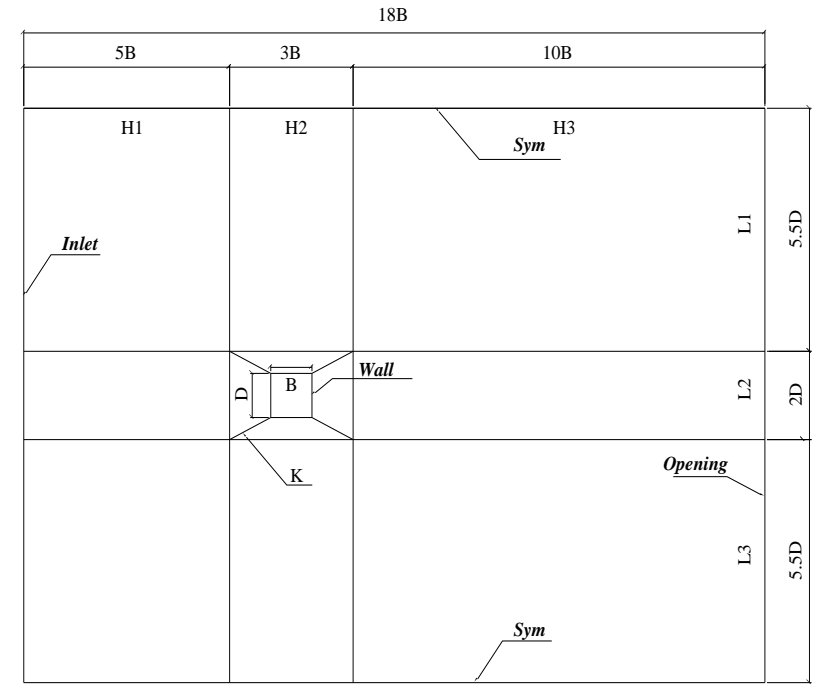

(b) Boundary condition

Fig. 2. Domain mesh and boundary conditions for 2D LES 
Z. Liu / JAFM, Vol. 3, No. 1, pp. 35-45, 2010.

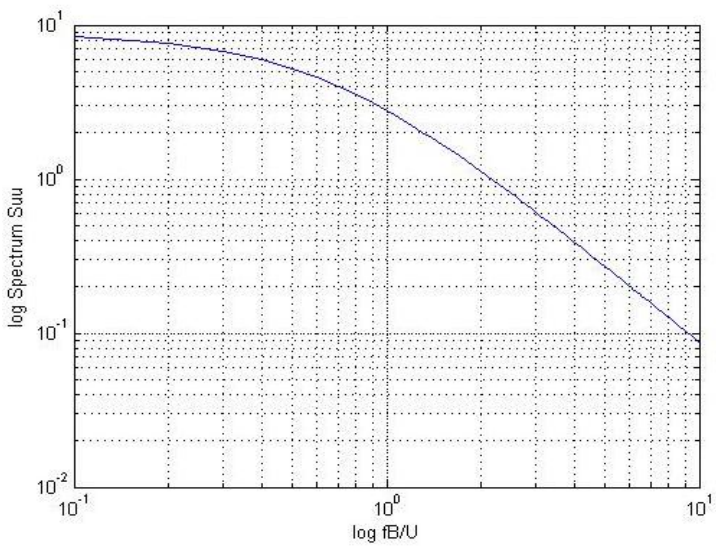

Fig. 3. Power spectrum u velocity

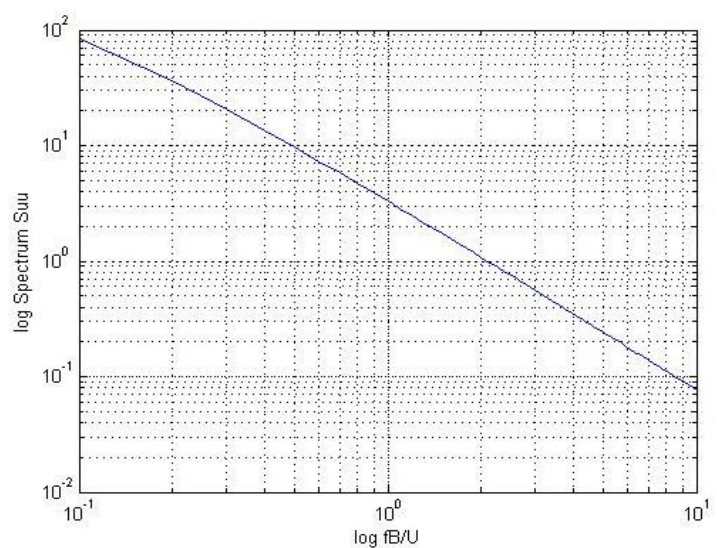

Fig. 4. Power spectrum w velocity
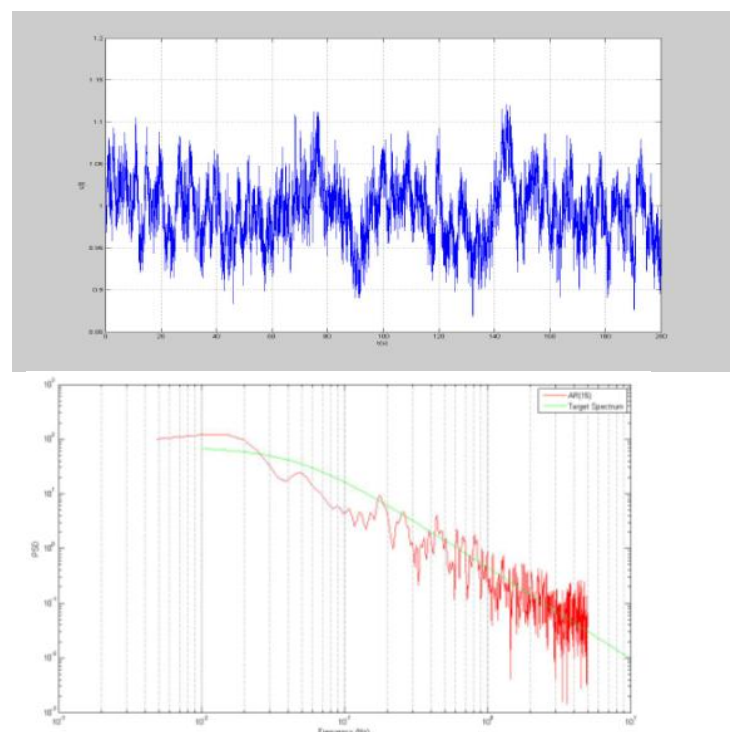

Fig. 5. Vertical distribution: time history and PSD of velocity in the bridge middle span
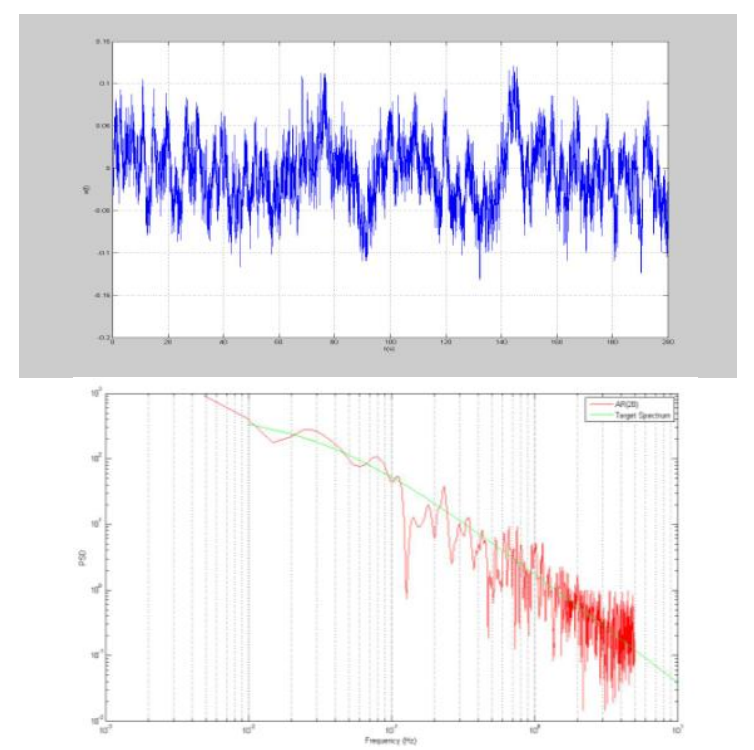

Fig. 6. Horizontal distribution: time history and PSD of velocity in the bridge middle span

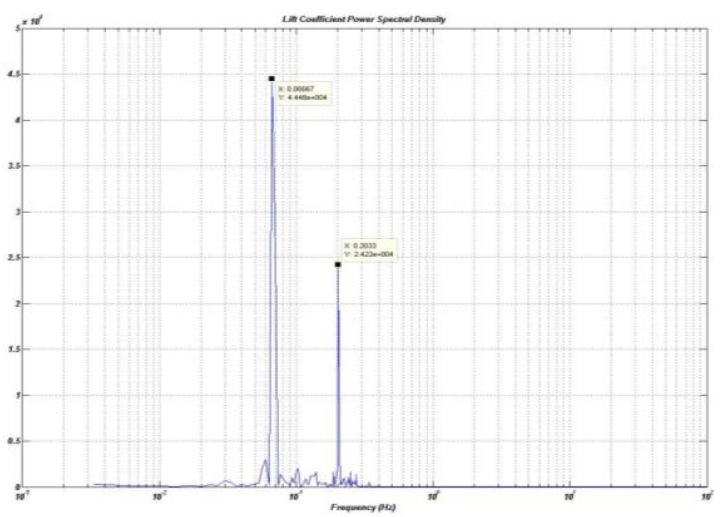

Fig. 7. R1 model lift coefficient PSD value

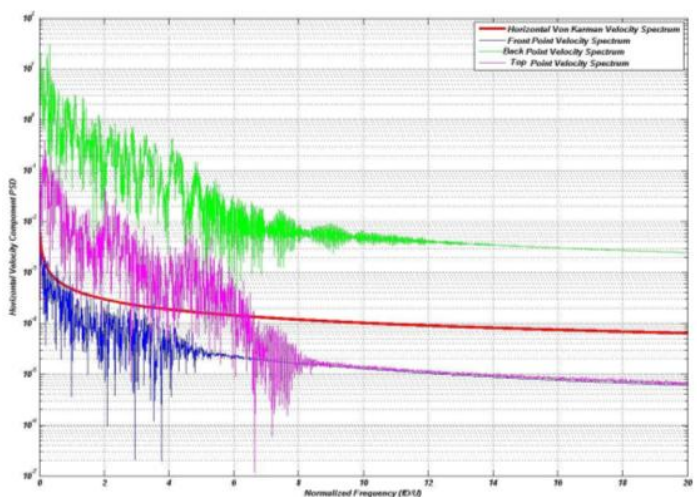

Fig. 8. Monitor points U velocity component PSD vs Von Karman velocity spectrum 


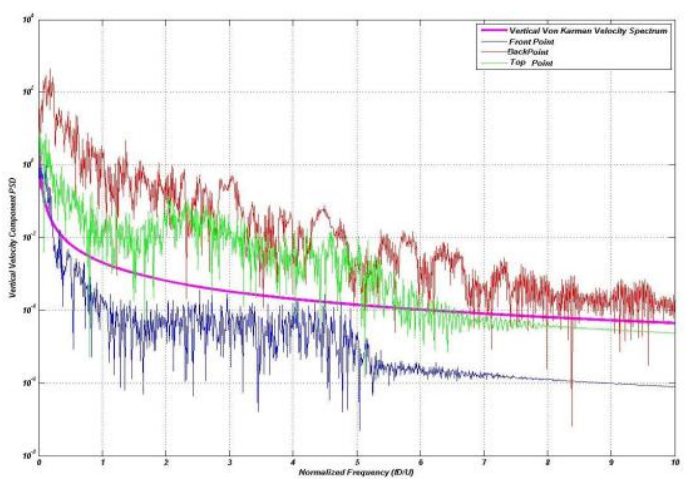

Fig. 9. Monitor points V velocity component PSD vs Von Karman velocity spectrum

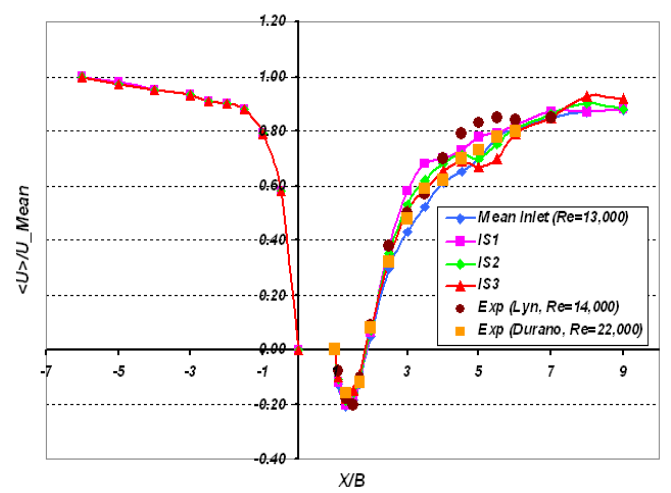

Fig. 10. Dimensionless velocity component time averaged value along the centre line

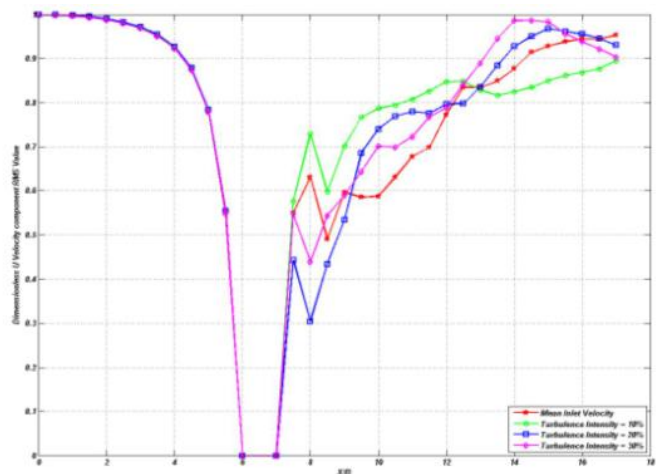

Fig. 11. Dimensionless velocity component RMS value along the centre line

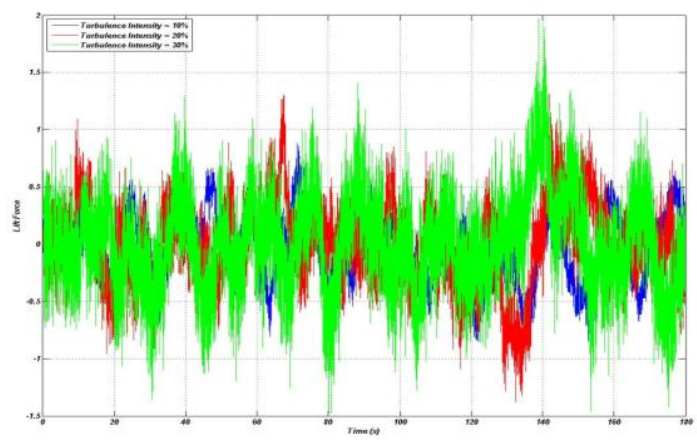

Fig. 12. Lift force time history curves under different inlet boundary conditions

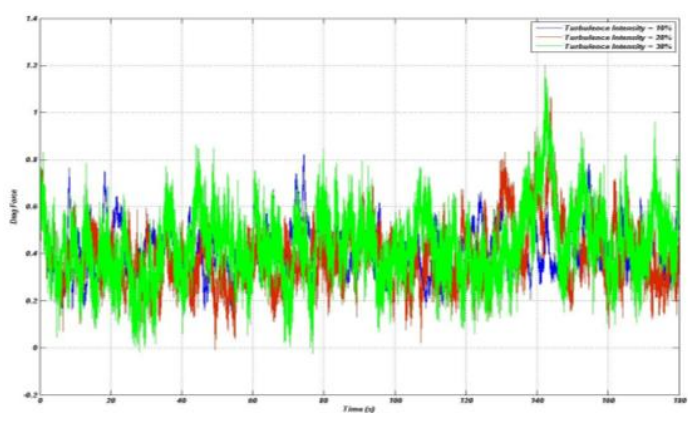

Fig. 13. Drag force time history curves under different inlet boundary conditions
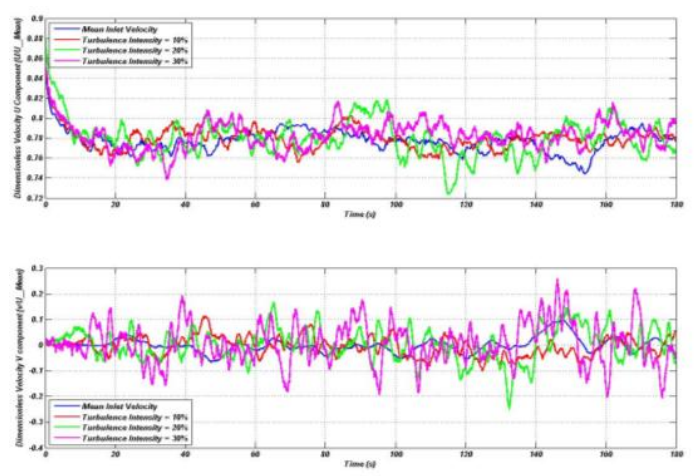

Fig. 14. Front point $\mathrm{u}$ and $\mathrm{v}$ velocity component time history curves

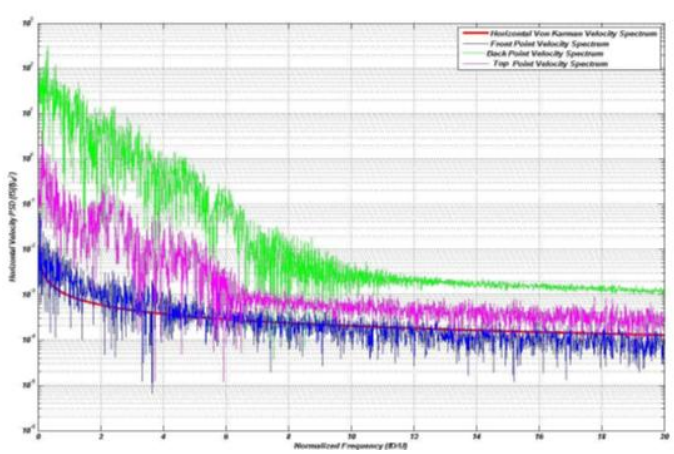

Fig. 15. U component velocity PSD under different position vs Van Karman spectrum

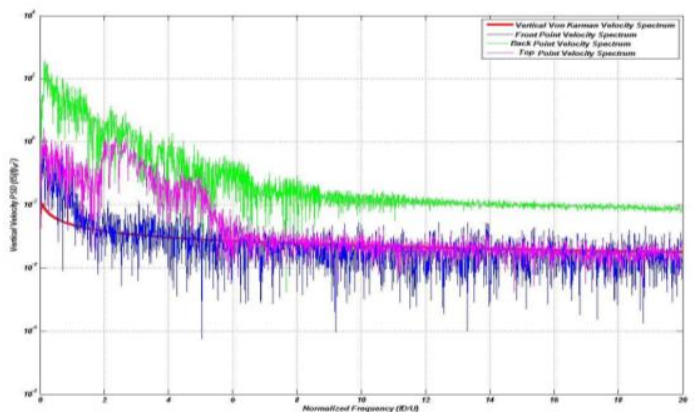

Fig. 16. V component velocity PSD under different position vs Van Karman spectrum 

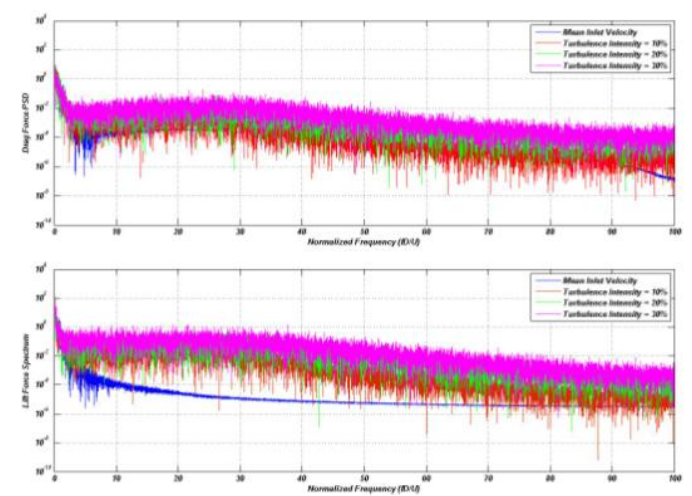

Fig. 17. Square cylinders lift and drag force PSD value under different inlet condition
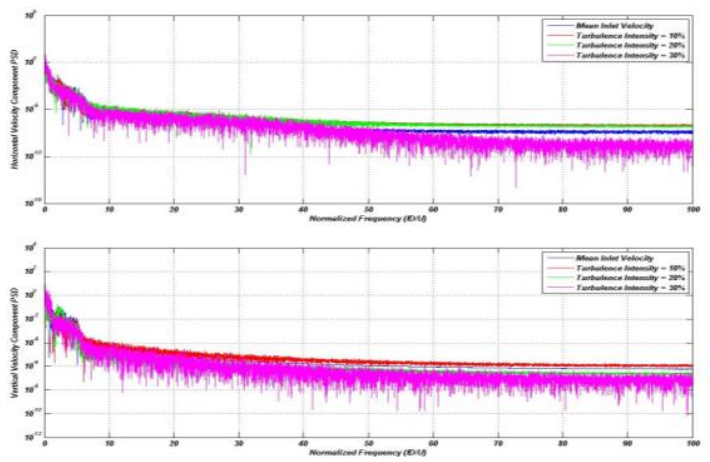

Fig. 18. Velocity component PSD value at top point
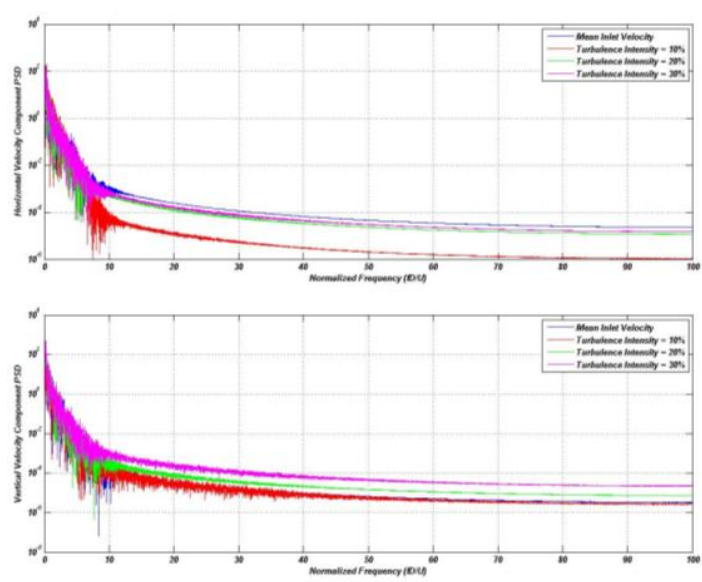

Fig. 19. Velocity Component PSD value at back point

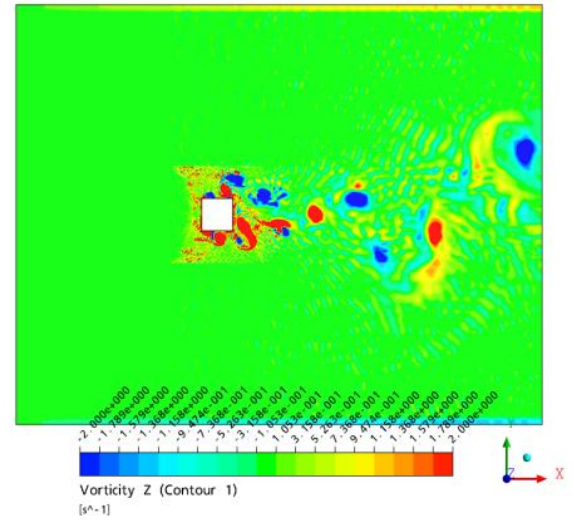

(a) Turbulence Intensity $=0$

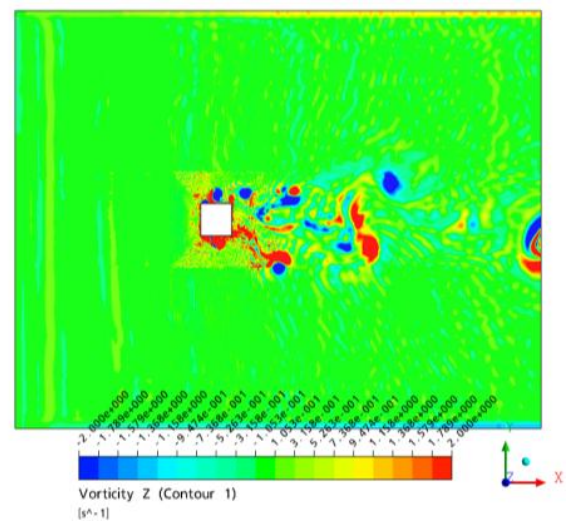

(b) Turbulence Intensity $=10 \%$

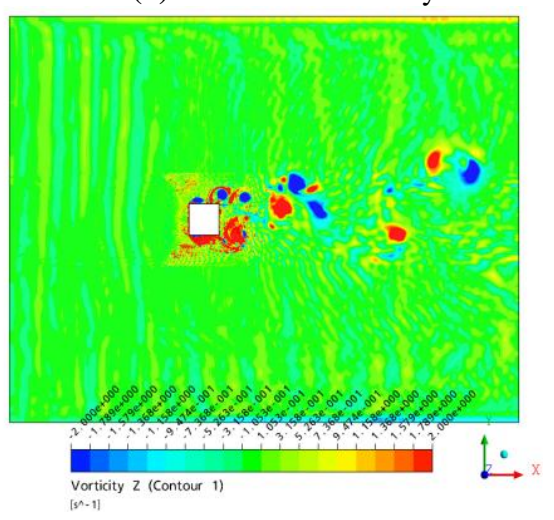

(a) Turbulence Intensity $=20 \%$

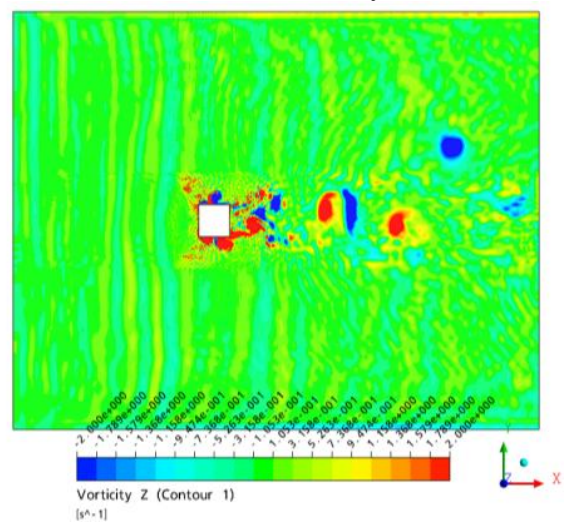

(b) Turbulence Intensity $=30 \%$

Fig. 20. Instantaneous vorticity $\mathrm{Z}$ at time $=120 \mathrm{~s}$ 